\title{
Expansion of Stodden et al.'s Model
}

\author{
Rodrigo A. Lima ${ }^{1} \bigotimes \cdot$ Clemens Drenowatz $^{2} \bigotimes \cdot$ Karin A. Pfeiffer ${ }^{3} \square$
}

Accepted: 14 December 2021 / Published online: 7 February 2022

(C) The Author(s), under exclusive licence to Springer Nature Switzerland AG 2022

\section{Introduction}

In 2008, Stodden et al. [1] published a framework model hypothesising the relationships among motor competence, physical activity, perceived motor competence, healthrelated fitness and weight status in children and adolescents. As a result, many subsequent investigations tested elements of the concepts depicted in the Stodden et al. [1] framework. Stodden et al.'s model was especially relevant for stimulating research investigating the importance of motor competence in relation to the other outcomes in the framework. Recently, Barnett et al. [2] conducted a systematic review to synthesise the longitudinal and experimental evidence around Stodden et al.'s model (with motor competence as a central point) in order to evaluate current evidence on the relationships among the proposed correlates of motor competence.

Although Stodden et al.'s model yielded a considerable number of investigations, there are multiple pathways with a limited number of studies [2]. From the 36 pathways evaluated, there were 18 pathways $(50 \%)$ without a sufficient number of studies (minimum of four studies) to draw conclusions, and five pathways presented indeterminate/inconsistent evidence. From the 13 remaining pathways, nine (22\% from 36 pathways) exhibited strong evidence. The other four pathways were not supported by the literature [2].

This commentary accompanies a systematic review published 31 August 2021.

This commentary refers to the article available at https://doi.org/ 10.1007/s40279-021-01516-8.

Rodrigo A. Lima

rodrigoantlima@gmail.com

1 Research, Innovation and Teaching Unit, Parc Sanitari Sant Joan de Déu, CIBERSAM, Sant Boi de Llobregat, Barcelona, Spain

2 Division of Sport, Physical Activity and Health, University of Education Upper Austria, Linz, Austria

3 Department of Kinesiology, Michigan State University, East Lansing, MI, USA
A complete synthesis is provided in Fig. 1, which is taken from Barnett et al.'s review [2] and allows for drawing the following conclusions:

1. The potential association of perceived competence with motor competence in Stodden et al.'s model has been overlooked, as none of these pathways were examined by a sufficient number of studies to draw conclusions. Only three longitudinal studies were included in the review conducted by Barnett et al. [2-5].

2. The pathways linking motor competence to physical activity are the most critical pathways to interpret. The direct pathways between motor competence and physical activity showed no or indeterminate evidence to support their relationship, except the pathway from composite motor competence to physical activity, which showed strong and positive evidence of the relationship. On the other hand, the reciprocal pathway between motor competence and physical activity mediated via health-related fitness exhibited strong evidence supporting it.

In addition to better assessment of physical activity and motor competence, it might be necessary to account for age group differences in this relationship as an increase in the strength of the relationship between motor competence and physical activity is expected with increasing age [1]. The importance of age in the motor competence-physical activity relationship needs to be better addressed.

3. The relationship from overall motor competence to health-related fitness was strong and positive whereas the reverse pathway was indeterminate. Considering only locomotor motor competence, rather than total motor competence, there was, however, strong evidence supporting the reciprocal pathway with health-related fitness. Nevertheless, there was an insufficient number of studies evaluating object control and composite constructs of motor competence in relation to health-related fitness.

4. The pathway between motor competence and weight status showed strong evidence supporting the relation- 


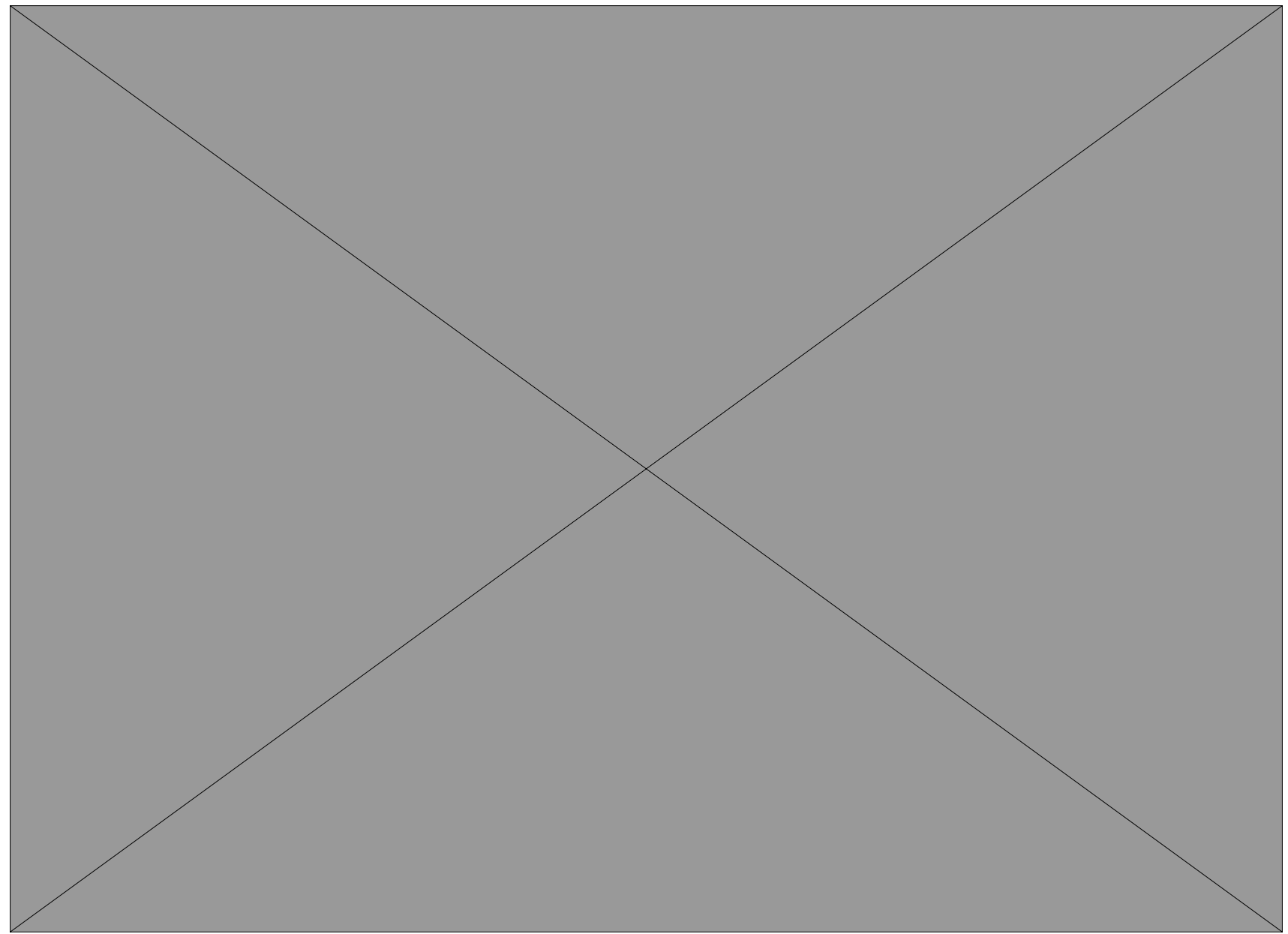

Fig. 1 Summarised longitudinal and experimental evidence around the Stodden et al. model from the study conducted by Barnett et al. [2]. Reprinted from Barnett et al. [2] with permission from Springer

ship in both directions despite the insufficient number of studies evaluating most of the pathways.

\section{Expanding the Stodden et al. Model}

Despite the need for further evaluation, the publication of Stodden et al.'s model yielded some relevant progress in the field. Frameworks such as the one from Stodden et al. [1] stimulate advances in the field and might be used as a starting point to evaluate the health of youth more holistically. In this light, we propose an extension of the Stodden et al. framework by adding three groups of health outcomes to the model.

It is not the purpose of this commentary to provide an evidence-based justification supporting the expansion of the Stodden et al. model. We simply gathered some evidence regarding important child and adolescent health outcomes, including metabolic health, mental health, and cognition and academic performance that might be linked to Stodden et al.'s framework. Based on the insights gained by Barnett et al. [2], the implementation of these additional variables may be a viable extension to Stodden et al.'s original model that could guide future research on children's and adolescents' health and well-being.

Figure 2 is a simplified representation of the extension of the Stodden et al. model that we are proposing. In summary, Blocks A and B are derived from the Stodden et al. model published in 2008 [1]. The dotted arrows between blocks of variables represent the expected relationship between all or at least most variables in the previous block with the following block of variables. As an illustration, besides the relationships between blocks A and B foreseen by Stodden et al. [1], we expect physical activity, health-related fitness, motor competence and weight status (variables in Block B) to be directly associated with the variables in the Block $\mathrm{C}$ (delimited by the dotted orange square). The predicted placement and relationship among variables in this proposed model (Fig. 2) are detailed below. 


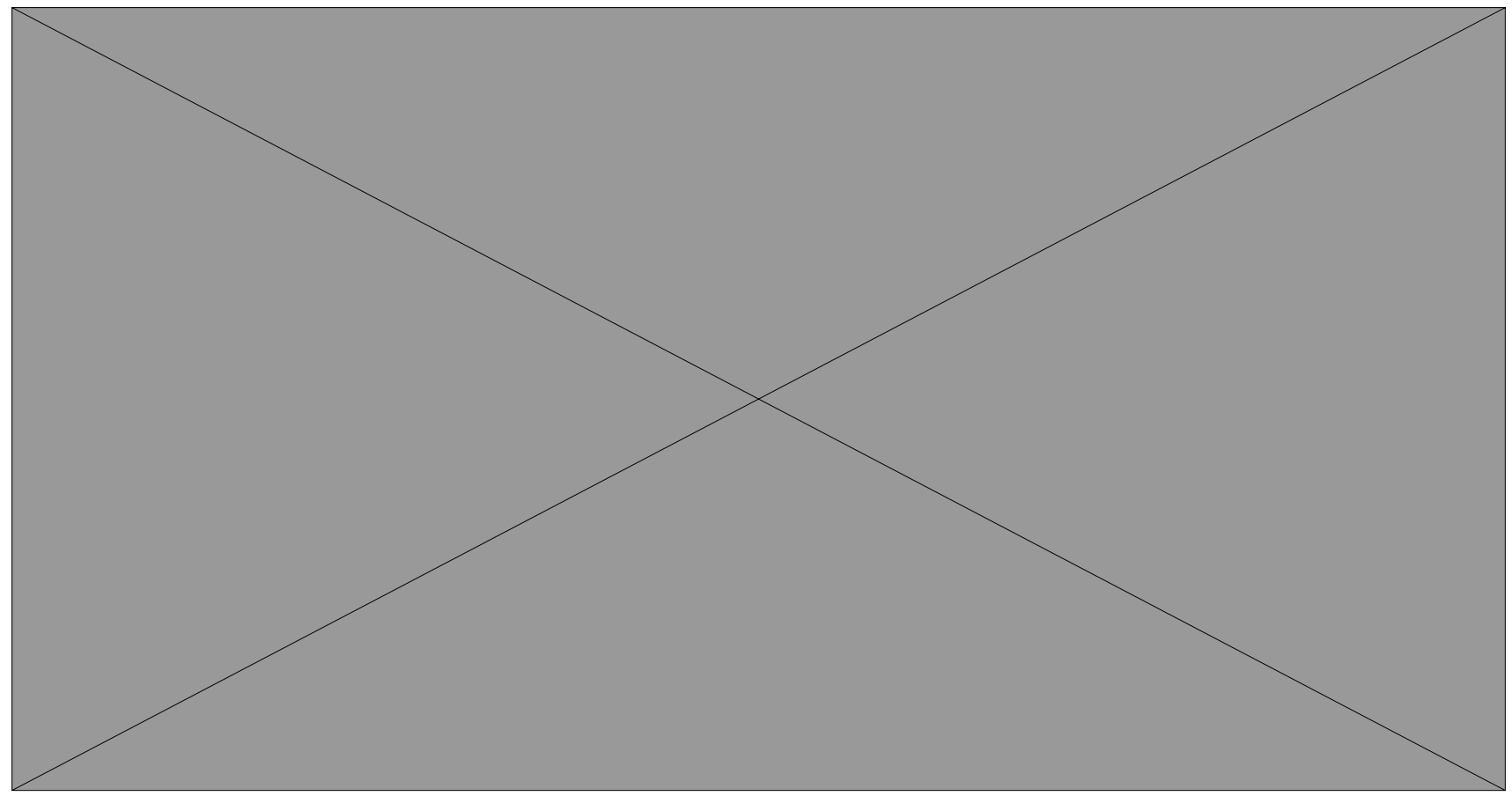

Fig. 2 Proposed extension of Stodden et al.'s model. Dotted arrows represent the relationship between one block of variables (denoted by the squares) in relation to the next block in the model. More specifically, note that all variables in the Block A (denoted by the light blue square) are assumed to be directly associated with weight sta-

\subsection{Metabolic Health}

From the group of outcomes first included in the Stodden et al. model, especially physical activity, health-related fitness and weight status have been linked to metabolic health (triglycerides, glucose, insulin, cholesterol, blood pressure, etc.) in children and adolescents [6-8]. Metabolic health might mediate the impact of increased physical activity, health-related fitness and motor competence in relation to weight status [9]. Moreover, metabolic health might also be a result of improved weight status because of changes in physical activity, health-related fitness and motor competence levels [10]. The 'placement' of metabolic health in the Stodden et al. model might be related to the age-group in focus. In younger children, it is expected that metabolic health is impacted by weight status, whereas metabolic health and weight status might present a more symbiotic relationship with increasing age.

\subsection{Cognition and Academic Performance}

A considerable number of studies have linked physical activity and health-related fitness to cognition and academic performance [11-13]. More recently, motor competence has been shown to be associated with cognition and academic tus. Moreover, physical activity, health-related fitness, motor competence and weight status (Block B) are expected to be directly associated with the variables in the block $\mathrm{C}$ (denoted by the dotted orange square)

performance [14]. Although understudied, physical activity, health-related fitness and motor competence are hypothesised to be related to cognition and academic performance via neurobiological, psychosocial and behavioural mechanisms $[15,16]$. We presume that physical activity, healthrelated fitness and motor competence may be associated with cognition and academic performance both directly and mediated via weight status.

\subsection{Mental Health}

Motor competence, physical activity, health-related fitness and weight status have been associated with mental health in children and adolescents [15, 17-21]. It is feared that the mental health of youth might deteriorate as a consequence of the Covid-19 pandemic. Therefore, it is of the highest priority to conduct original studies investigating the role of the Stodden et al. model in relation to child and adolescent mental health. The causal mechanisms linking the variables in the Stodden et al. [1] model with mental health are still unclear, but the few studies there are, indicate the importance of neurobiological, psychosocial and behavioural mechanisms $[15,22]$. There is evidence supporting the inclusion of mental health after weight status in the Stodden et al. model [15, 17-21], although mental health has 
also been shown to be mutually related to weight status, especially during adolescence [23].

\section{Conclusions}

We urge the scientific community to evaluate the Stodden et al. [1] model in depth. Longitudinal work, especially experimental research using different doses of interventions, is needed. Priority should be given to studies with longerterm follow-ups and multiple monitoring periods since the progression of the associations between pathways across time is still under-evaluated. The developmental nature of the relationships is a critical element in the Stodden et al. model that needs further analysis. Moreover, we encourage experimental studies to evaluate the possible causal chain of the associations among motor competence, perceived motor competence, physical activity and health-related fitness with metabolic health, mental health, and cognition and academic performance, including as many components from Stodden et al.'s [1] original model as possible.

\section{Declarations}

Funding No funding was received for this study.

Conflict of interest None to declare.

Availability of data and material Not applicable.

Code availability Not applicable.

Author contributions RAL drafted the first version of the manuscript. All authors critically revised and drafted the manuscript.

Ethics approval Not applicable.

Consent to participate Not applicable.

Consent for publication Not applicable.

\section{References}

1. Stodden DF, Goodway JD, Langendorfer SJ, Roberton MA, Rudisill ME, Garcia C, et al. A developmental perspective on the role of motor skill competence in physical activity: an emergent relationship. Quest. 2008;60:290-306.

2. Barnett L, Webster E, Hulteen R, De Meester A, Valentini N, Lenoir M, et al. Through the looking glass: a systematic review of longitudinal evidence, providing new insight for motor competence and health. Sports Med. 2021. https://doi.org/10.1007/ s40279-021-01516-8.

3. Marouli A, Papavasileiou G-E, Dania A, Venetsanou F. Effect of a psychomotor program on the motor proficiency and self-perceptions of preschool children. J Phys Educ Sport. 2016;16:1365-71.
4. Lander N, Mergen J, Morgan P, Salmon J, Barnett L. Can a teacher-led RCT improve adolescent girls' physical self-perception and perceived motor competence? J Sports Sci. 2019;37:357-63.

5. Lloyd M, Saunders T, Bremer E, Tremblay M. Long-term importance of fundamental motor skills: a 20-year follow-up study. Adapt Phys Act Q. 2014;31:67-78.

6. Whooten R, Kerem L, Stanley T. Physical activity in adolescents and children and relationship to metabolic health. Curr Opin Endocrinol Diabetes Obes. 2019;26:25-31.

7. Mintjens S, Menting MD, Daams JG, van Poppel MNM, Roseboom TJ, Gemke RJBJ. Cardiorespiratory fitness in childhood and adolescence affects future cardiovascular risk factors: a systematic review of longitudinal studies. Sport Med. 2018;48:2577-605.

8. García-Hermoso A, Ramírez-Campillo R, Izquierdo M. Is muscular fitness associated with future health benefits in children and adolescents? A systematic review and meta-analysis of longitudinal studies. Sport Med. 2019;49:1079-94. https://doi.org/10.1007/ s40279-019-01098-6.

9. Lima RA, Andersen LB, Soares FC, Kriemler S. The causal pathway effects of a physical activity intervention on adiposity in children: The KISS Study cluster randomised clinical trial. Scand J Med Sci Sports. 2020;30:1685-91.

10. Díez-Fernández A, Sánchez-López M, Mora-Rodríguez R, Notario-Pacheco B, Torrijos- Niño C, Martínez-Vizcaíno V. Obesity as a mediator of the inf luence of cardiorespiratory fitness on cardiometabolic risk: a mediation analysis. Diabetes Care. 2014;37:855-62. https://doi.org/10.2337/dc13-0416/-/DC1.

11. Santana CCA, Azevedo LB, Cattuzzo MT, Hill JO, Andrade LP, Prado WL. Physical fitness and academic performance in youth: a systematic review. Scand J Med Sci Sports. 2017;27:579-603. https://doi.org/10.1111/sms.12773.

12. de Greeff JW, Bosker RJ, Oosterlaan J, Visscher C, Hartman E. Effects of physical activity on executive functions, attention and academic performance in preadolescent children: a meta-analysis. J Sci Med Sport. 2018;21:501-7.

13. Marques A, Santos DA, Hillman CH, Sardinha LB. How does academic achievement relate to cardiorespiratory fitness, selfreported physical activity and objectively reported physical activity: a systematic review in children and adolescents aged 6-18 years. Br J Sports Med. 2018;52:1039.

14. Macdonald K, Milne N, Orr R, Pope R. Relationships between motor proficiency and academic performance in mathematics and reading in school-aged children and adolescents: a systematic review. Int J Environ Res Public Health. 2018;15:1603.

15. Lubans D, Richards J, Hillman C, Faulkner G, Beauchamp M, Nilsson M, et al. Physical activity for cognitive and mental health in youth: a systematic review of mechanisms. Pediatrics. 2016;138:e20161642-e20161642. https://doi.org/10.1542/peds. 2016-1642.

16. Voss MW, Vivar C, Kramer AF, van Praag H. Bridging animal and human models of exercise-induced brain plasticity. Trends Cogn Sci. 2013;17:525-44.

17. Rodriguez-Ayllon M, Cadenas-Sánchez C, Estévez-López F, Muñoz NE, Mora-Gonzalez J, Migueles JH, et al. Role of physical activity and sedentary behavior in the mental health of preschoolers, children and adolescents: a systematic review and metaanalysis. Sport Med. 2019;49:1383-410.

18. Sutaria S, Devakumar D, Yasuda SS, Das S, Saxena S. Is obesity associated with depression in children? Systematic review and meta-analysis. Arch Dis Child. 2019;104:64-74.

19. Korczak DJ, Madigan S, Colasanto M. Children's physical activity and depression: a meta-analysis. Pediatrics. 2017. www.aappu blications.org/news. Accessed 17 Sept 2021.

20. Gu X, Zhang T, Chu TL, Keller MJ, Zhang X. The direct and indirect effects of motor competence on adolescents' 
mental health through health-related physical fitness. J Sports Sci. 2019;37:1927-33.

21. Redondo-Tebar A, Fatouros IG, Martinez-Vizcaino V, RuízHermosa A, Notario-Pacheco B, Sanchez-Lopez M. Association between gross motor competence and health-related quality of life in (pre)schoolchildren: the mediating role of cardiorespiratory fitness. Routledge. 2020;26:51-64. https://doi.org/10.1080/17408 989.2020.1800618.
22. Kandola A, Ashdown-Franks G, Hendrikse J, Sabiston CM, Stubbs B. Physical activity and depression: towards understanding the antidepressant mechanisms of physical activity. Neurosci Biobehav Rev Pergamon. 2019;107:525-39.

23. Mannan M, Mamun A, Doi S, Clavarino A. Prospective associations between depression and obesity for adolescent males and females - a systematic review and meta-analysis of longitudinal studies. Homberg J, editor. PLoS ONE. 2016;11: e0157240. 